Université กn
de Montréal

Serge Sultan, Université de Montréal

How to cite this article:

Aramideh, J., Ogez, D., Mizrahi, T., Charest, M. C., Plante, C., Duval, M., \& Sultan, S. (2020). Do professionals change their communication behaviours following a training in hypnosis-derived communication? A feasibility study in pediatric oncology. Complementary Therapies in Medicine, 102426.

https://doi.org/10.1016/j.ctim.2020.102426

DOI: $\quad$ https://doi.org/10.1016/j.ctim.2020.102426

PMID: $\quad 32951704$ 


\section{Do professionals change their communication behaviours following a training in hypnosis- derived communication? A feasibility study in pediatric oncology}

Jennifer Aramideh, M.Sc. ${ }^{\mathrm{a}, \mathrm{b}}$, David Ogez, Ph.D. ${ }^{\mathrm{a}, \mathrm{b}}$, Terry Mizrahi, M.D. ${ }^{\mathrm{a}}$, Marie-Claude Charest, M.Ps. ${ }^{\mathrm{a}}$, Caroline Plante, B.Sc.N. ${ }^{\mathrm{a}}$, Michel Duval, M.D. ${ }^{\mathrm{a}, \mathrm{c}} \&$ Serge Sultan, Ph.D. ${ }^{\mathrm{a}, \mathrm{b}, \mathrm{c}^{*}}$

a Department of Hematology-Oncology, CHU Sainte-Justine, 3175, Chemin de la Côte-SainteCatherine, Montréal, Québec, Canada, H3T 1C5

${ }^{\mathrm{b}}$ Department of Psychology, Université de Montréal, Pavillon Marie-Victorin PO Box. 6128, Succursale Centre-ville, Montréal, Québec, Canada, H3C 3J7

c Department of Pediatrics, Université de Montréal, 3175, Chemin de la Côte-Sainte-Catherine, Montréal, Québec, Canada, H3T 1C5

\section{*Corresponding author:}

Name: Serge Sultan, Ph.D.

Address: CHU Sainte-Justine, 3175 Chemin de la Côte-Sainte-Catherine, Montréal, Québec, Canada, H3T 1C5

Telephone: (514) 345-4931 \#7068

Email: serge.sultan@umontreal.ca

\section{Funding}

Support for this research came from the Sainte-Justine UHC Foundation through the Centre of Psycho-Oncology (recipient Serge Sultan) and the Fonds pour la Recherche du Québec - Santé (FRQs) through a post-doctoral fellowship to David Ogez.

Word count: 3289 words 


\section{$1 \quad \underline{\text { Abstract }}$}

2 Objectives: The purpose of this study was to test the feasibility of a training in hypnotic

3 communication techniques (HCTech) for pediatric nurses to prevent procedural pain and distress

4 in children during venipunctures. Specifically, this study aimed to (1) assess nurses' mastery of

5 HCTech and (2) nurses' experience regarding the training program. Methods: Participants were 6

6 female pediatric nurses and 33 of their cancer patients. Nurses took part in a 4-day theoretical and

7 practical training in HCTech. Venipuncture procedures were video-recorded and assessed to

8 evaluate nurses' mastery of HCTech using a standardized scale. Pre-training use of HCTech was

9 compared with post-training and follow-up for the entire nurse sample and across nurses with the

10 same patients (109 nurse-patient interactions). After the follow-up, nurses were questioned about

11 their experience in regards to the training and activities (themes and practice). Results: Results

12 showed medium pre-post changes in hypnotic communication behaviours (pre-post $d=0.74$ ), with

13 changes maintaining at follow-up (pre-follow-up $d=0.97$ ). Interviews transcripts' analyses revealed

14 moderate levels of motivation and satisfaction regarding the training content and format. Nurses

15 suggested to emphasize on the practice of HCTech in a noisy outpatient clinic as well as offer more

16 practical exercises. Conclusion: A 4-day training in hypnotic communication techniques translated

17 into the use of HCTech by nurses practicing in pediatric oncology when comparing the same dyads

18 at baseline, post-training and follow-up. Results support further refinement and suggest nurses

19 could be trained to prevent pain and distress with hypnosis-derived communication strategies.

21 Key words:

22 Hypnosis-derived communication, Healthcare professionals, Nurses, Pediatrics, Oncology,

23 Venipunctures, Pain, Procedural distress 


\section{$24 \quad$ 1. Introduction}

25 Pediatric oncology patients undergo frequent painful needle procedures during the course

26 of treatment, which are associated with important levels of pain and distress. ${ }^{1}$ Unmanaged pain can

27 result in several physiological and psychological negative long-term consequences in children. ${ }^{2}$

28 Pediatric cancer survivors may develop long-term medical traumatic stress, partly caused by

29 medical procedures. ${ }^{3}$ It is therefore crucial to provide early pain and distress management in

30 pediatric settings.

31 Latest developments in pediatric pain management have shown that different types of

32 interventions are effective in decreasing children's pain and distress when undergoing various

33 medical procedures: pharmacological interventions (e.g. local anesthetic such as EMLA cream $\AA^{4}$ )

34 and non-pharmacological interventions (e.g. Buzzy ${ }^{5}$, distraction ${ }^{6}$, hypnosis ${ }^{1,7}$ ). Among these,

35 hypnotic communication involves the use of communication techniques derived from medical

36 hypnosis. In healthcare, hypnotic communication techniques (HCTech) can be used as a single

37 intervention or as an adjunct intervention with other physical and pharmacological pain

38 management methods. ${ }^{8}$ Several studies have shown that the use of hypnosis and hypnosis-derived

39 communication, as a single or combined intervention, can be effective in decreasing pediatric

40 cancer patients' procedural pain ${ }^{9-19}$ and distress ${ }^{9,12-17}$. In these studies, healthcare professionals

41 performed the medical procedure while another professional, a hypnotherapist, used hypnosis

42 strategies with patients. For practical reasons and cost issues, it would be beneficial if nurses

43 themselves used HCTech while performing medical procedures. Currently, hypnosis and HCTech

44 applied by nurses are underutilized in the healthcare system, partially due to a lack of formalized

45 professional training. ${ }^{20}$ Notably, no study has yet systematically assessed the effects of HCTech

46 training on actual practice.

47 This feasibility study aimed to assess a basic HCTech training for pediatric oncology 
48 nurses. Specifically, this study aimed to assess (1) changes in nurses use of HCTech in clinical

49 practice and (2) nurses' experience regarding the training program. It is essential to document how

50 trainees' behaviours change following a training before studying possible translation to patient

51 outcomes in order to link possible favorable changes with the effective use of HCTech.

\section{2. Methods}

The study was conducted at Sainte-Justine University Hospital Centre's (Sainte-Justine

55 UHC) Hematology-Oncology daycare clinic (Montreal, Quebec, Canada). The study was approved

56 by the Sainte-Justine UHC Research Ethics Committee and all participants, nurses and patients,

57 provided written informed consent.

\section{$58 \quad$ 2.1. Inclusion and Exclusion Criteria}

To be eligible, nurses had to work at the outpatient hematology-oncology daycare clinic,

60 have previous experience performing venipunctures (VPs) in pediatrics and have no prior

61 experience in hypnosis or hypnosis-derived communication. Patients had to be aged between 5 and

6218 years old, have regular follow-ups at the clinic and understand French. Patients who had been

63 previously exposed to hypnosis or hypnosis-derived communication were excluded, as were those

64 who came at the clinic for an unexpected appointment (e.g. emergency) and those with a psychiatric

65 disorder, as documented in medical charts.

\section{2.2. Participants and Setting}

Six nurses and 36 patients were solicited to participate in this study. During a meeting

68 between the research team (JA and TM) and the Sainte-Justine UHC's oncology daycare clinic

69 nursing staff, the study protocol was presented to all practicing nurses. Following this meeting, six

70 nurses volunteered to develop their skills to reduce their patients' pain and distress using this

71 program and were included in the study. Therefore, all nurses agreed to participate in this research 
72 study. Using a convenience sampling method, each nurse was assigned 6 consecutive patients from

73 the clinic's computer database by a research assistant with no prior selection. Once the first six

74 patients meeting the criteria were identified, they were contacted by phone and given preliminary

75 information on the study. Patients and their parent(s) subsequently met with a researcher (TM or

76 JA) to receive additional information about the study and sign consent. Three patients and their

77 parent(s) declined to participate. Following their inclusion, patients were received at the

78 hematology-oncology daycare clinic by the nurses who performed VPs, which were video-

79 recorded and assessed by the research team.

\section{2.3. Hypnotic Communication Techniques Training}

81 The training consisted in four sessions that were conducted at Sainte-Justine UHC's cancer

82 care centre by a hypnosis-certified psychologist (MCC). The training and all communication

83 techniques presented were adapted from a hypnotic suggestions reference guide ${ }^{21}$ and a book on

84 the practice of hypnosis in pediatrics ${ }^{22}$. Each session lasted approximately five hours and

85 emphasized on theoretical components and practical exercises. Session 1 focused on the

86 identification of pediatric pain and the use of hypnosis to manage procedural pain and distress, the

87 basics of clinical hypnosis, the differences between Ericksonian and clinical hypnosis, and the use

88 of hypnosis within the health field, including the ethical challenges of this practice with children.

89 Session 2 focused on methods and techniques for pediatric pain management, highlighting the

90 importance of the nurse-patient relationship, the language to use when accompanying patients

91 during a hypnotic intervention, differences between distraction and clinical hypnosis-derived

92 communication techniques. This session also included notions about the development of hypnotic

93 state, pain-relief suggestions and post-hypnotic suggestions as well as concepts of basic

94 pain/distress directed strategies. Nurses took part in practical exercises. In turns, they tried different

95 techniques: deep breathing, conversational hypnosis, sensation changes suggestions (e.g. changes 
96 in perception), the magic glove, etc. ${ }^{21,22}$ Session 3 focused on practical exercises during which

97 nurses role-played and received feedback from the trainer. Additional techniques were put into

98 practice: the switch, pain transformation, the bubble, guided imagery (e.g. preferred place), etc. ${ }^{21,22}$

99 Detailed definitions and examples of hypnotic techniques are available in a supplementary file.

100 Session 4 focused on supervising the acquired techniques and improving their technical and

101 relational skills. Nurses were supervised for two encounters and were given feedback. A final group

102 session was organized to alleviate barriers and implementation difficulties (see ${ }^{23}$ for additional

103 details).

104 2.4. Assessments

105 Nurses' communication behaviours were assessed at four time-points with the same patient:

106 two pre-training (T1 and T2 occurring in average respectively 148 and 119 days before the training)

107 and two post-training (T3 and T4 occurring in average respectively 137 and 203 days after the

108 training). The training occurred in September 2015 and data collection spanned from March 2015

109 to November 2016. Two pre-training assessments were included to control for natural evolution

110 over time and measurement error. Although a number of three data points is usually recommended

111 to determine the baseline ${ }^{24}$, this was not organisable in practice and only two measures were taken.

\section{$112 \quad$ 2.5. Measures}

113 Sainte-Justine Hypnotic Communication Assessment Scale (SJ-HCAS): This scale was used

114 to assess communication behaviours in video-recorded nurse-patient encounters. ${ }^{23}$ It is based on

11511 core items of hypnosis-derived communication to prevent pain and distress in children. For each

116 item, an independent rater evaluates whether the behaviour is present (1) or absent (0). Two count

117 scores are computed, one on the quality of the relationship (subscore 0 to 5) and one on the quality

118 of the communication technique (subscore 0 to 6 ). A total score is computed by adding all 11 items

119 (range 0-11). For comparison purposes, each score was transformed to a percentage in the present 
120 study. Previous analyses have demonstrated excellent inter-rater reliability for the total score and

121 the two subscores (median ICC $=0.879$ ), including when raters were blind of assessment time-

122 points and when raters had different professional backgrounds. ${ }^{23}$

123 Interviews: At the end of the study, brief semi-structured interviews were conducted with

124 each nurse to collect feedback on their: (1) initial reasons and levels of motivation to participate in

125 the training (10-point Likert scale : $0=$ not motivated $-10=$ very motivated), (2) use of HCTech

126 in daily practice, (3) perceived benefits of the training, (4) training satisfaction (10-point Likert

127 scale : $0=$ not satisfied - $10=$ very satisfied), and training assessment (positive and negative

128 components) and (5) recommendations on possible improvements for future training.

\section{2.6. Statistical Analyses}

130 To explore consistency within the two baseline time-points, paired sample t-tests and 131 Pearson correlations for each score and subscore of the SJ-HCAS were used. Two-way random

132 absolute stability ICCs were also computed and interpreted as 0-.40 = poor, .40-.59= fair, .60-.74

$133=$ good, .75 to $1.0=$ excellent. $^{25}$ As this supported minimal change and strong consistency, both

134 pre-training time-points were averaged into a unique baseline value. To evaluate changes

135 associated with the training, Wilcoxon signed-rank non-parametric tests were used. Difference

136 effect sizes were also computed for pre-post and pre-follow-up comparisons (Cohen's $d$ ). A

137 thematic analysis was performed on the qualitative data from satisfaction interviews. ${ }^{26}$ All

138 quantitative analyses were conducted using IBM SPSS Statistics 24 and, where appropriate, a

139 significance alpha threshold of 0.05 was used.

141 3. Results

142 3.1. Participants

143 Six female pediatric oncology nurses (aged 27-44), and 33 of their cancer patients (16 boys, 
14417 girls) aged $10 \pm 4$ years took part in this study. During the course of the feasibility study, 1 nurse

145 (Nurse E) went on maternity leave and was unable to complete the training as well as both post-

146 training time-points. Two patients passed away and 5 patients dropped out. For ethical reasons, we

147 offered Nurse E's 4 patients the hypnotic intervention as initially intended, but with the other nurses

148 being involved with them. However, as the research design is based on the follow-up of the same

149 nurse-patient dyads over time, these 4 patients were excluded from analysis. Across the four time-

150 points, 117 nurse-patient interactions were video-recorded and available for hypnotic

151 communication assessments. However, when accounting for dropouts and exclusions for analyses

152 purposes, pre-training use of HCTech was compared with post-training (5 nurses and 24 of their

153 patients) and follow-up (5 nurses and 22 of their patients) in 109 interactions (Flow chart on Figure

$1541)$.

155 3.2. Evolution of the use of HCTech across time-points

156 3.2.1. Baseline Levels

157 Baseline measures were stable in regards to the total score as well as the relationship and 158 technique subscores (ICCs $=0.630-0.766, d=-0.141-0.167)$. Consequently, we averaged these

159 time-points into a baseline score for each quantitative measure.

160 3.2.2. Nurses' mastery of hypnotic communication techniques

161 In regards to the entire nurse sample, an increased use HCTech was found in post training $(Z$

$162=-3.138, \mathrm{p}=0.002, d=0.74)$. This was reflected in an increased use of relationships strategies $(\mathrm{Z}$

$163=-2.942, \mathrm{p}=0.003, \mathrm{~d}=0.70)$ and techniques $(\mathrm{Z}=-2.710, \mathrm{p}=0.007, d=0.61)$. For all measures,

164 the post-training levels maintained at follow-up. Pre-follow-up effect sizes were medium-large for

165 the SJ-HCAS total score $(\mathrm{Z}=-3.614, \mathrm{p}<0.001, d=0.97)$ and for both the relationship $(\mathrm{Z}=-3.235$,

$166 \mathrm{p}=0.001, d=0.92)$ and technique subscores $(\mathrm{Z}=-2.976, \mathrm{p}=0.003, d=0.80)$. (Table 1$)$. A stability

167 between post and follow-up data points was observed for the entire nurse sample (total score: $\mathrm{Z}=$ 
$168-0.309, \mathrm{p}=0.757, d=-0.06$; relationship subscore: $\mathrm{Z}=-0.707, \mathrm{p}=0.480, d=-0.15$; technique

169 subscore: $\mathrm{Z}=-0.159, \mathrm{p}=0.873, d=0.00)$.

170 However, it is probable that differential evolution across nurses were aggregated in this overall

171 pattern. We used graphical displays illustrating pre-post-follow-up changes in hypnotic

172 communication techniques across nurses to explore this further (Figure 2). The overall pattern

173 visually emerging from these figures is that following the training, nurses mastered relational and

174 technical hypnotic communication skills and that these competencies were maintained over time.

175 However, nurses $\mathrm{C}$ and $\mathrm{D}$ experienced a larger increase in their hypnotic communication

176 behaviours. To explore this phenomenon, $d$ values were computed at the nurses level and

177 represented graphically (Figure 3). The results were consistent with Figure 2 and suggested larger

178 changes for nurses C and D while medium-small changes for nurses A, B and F. A supplementary

179 table providing all Wilcoxon signed-rank non-parametric tests results and $\mathrm{p}$ values is available

180 (Table S1).

\section{3.3. Nurses' Experience}

182 When describing quantitative scores of nurses' motivation and satisfaction, the nurses who

183 participated in the training reported being moderately motivated to take part in the training $(7.4 / 10$

$184 \pm 2.07)$ and expressed two motivational aspects: to better help patients and a curiosity about the

185 hypnotic technique (Table S2). Importantly, nurses were only moderately satisfied with the training $186(6 / 10 \pm 1.41)$.

187 When exploring nurses' experience qualitatively, only 3/5 nurses reported observing 188 changes in their practice after the training. Regarding the perceived benefits of using HCTech with 189 patients, 4 nurses (A, C, D and F) reported less anxiety in children during the painful needle 190 procedure. Two nurses (A and C) reported using more "distraction techniques" when performing 
191 VPs. Nurse F expressed that the training showed her different ways to interact with patients. Nurse

192 D specified that these benefits depended on the techniques used. In contrast to her colleagues, nurse

193 B mentioned that HCTech finally did not interest her also stating that some patients are simply not

194 sensitive to HCTech.

195

Personal benefits of using HCTech were only reported by one nurse (F) who experienced a

196 decrease in stress when performing VPs as a result of the training. Overall, a mixed picture

197 emerged, with all nurses reporting moderate levels of motivation and satisfaction with a significant

198 subset experiencing changes in their practice.

$199 \quad$ Nurses' qualitative training assessment also highlighted positive and negative components

200 (Table S2). Positive components can be summarized as: learning different types of techniques,

201 practicing among participants (role-play) and using of concrete situations. Negative components

202 were that some parts of the training seemed insufficiently articulated with practice or not

203 representative of situations encountered in the hematology-oncology daycare clinic. For example,

204 nurse B claimed that the daycare clinic's noisy environment was not conducive to this type of

205 intervention (see Table S2 for a detailed account of verbal responses). Nurses proposed two main

206 avenues to further refine this training. Firstly, the training should emphasize on more concrete

207 situations that are encountered in a day-to-day clinical practice. They considered that it would be

208 useful to produce video recordings more representative of outpatient clinics for modeling, 209 rehearsing and practicing intensively the techniques as part of the training itself. These recordings

210 would illustrate precisely how nurses use HCTech while performing medical procedures. Secondly,

211 one nurse proposed to better target professionals sensitive to non-pharmacological interventions.

212

\section{4. Discussion}

214 This study aimed to test the feasibility of a training in hypnotic communication for pediatric 
215 nurses, designed to prevent pain and distress in children undergoing painful procedures. The study

216 aimed to assess changes in the use of hypnotic communication techniques as well as nurses'

217 experience regarding the training program. The results showed for the first time that it is feasible

218 to train pediatric nurses in hypnotic communication for procedural pain and distress management

219 and that the training is acceptable and mostly positively assessed by nurses.

220 Results showed that after a brief training in hypnotic communication of approximately

221 twenty hours, pediatric nurses had a good mastery of HCTech and that newly acquired skills were

222 globally maintained over time.

223 Nurses' general mastery of HCTech can be understood at different levels, as these skills

224 may be both relational and technical. In regards to relational skills, the study showed an overall

225 increased use of relation strategies by nurses following the training. Considering that the

226 establishment of a good therapeutic relationship with the patient is a prerequisite for the use of

227 hypnosis-derived techniques in pediatrics ${ }^{27}$, the training seemed to allow nurses to become

228 sensitive to the importance of relational dimensions. It is also possible that nurses' previous

229 professional experience caring for sick children facilitated the integration of these hypnotic

230 relational skills in their daily practice. Concerning technical skills, the study equally showed an

231 increased use of technical hypnotic components following the training as demonstrated by the

232 objective assessments of communication behaviours. As the adaptation of the hypnotic technique

233 to the child is the second prerequisite for the use of hypnosis in pediatrics ${ }^{27}$, the training probably

234 allowed nurses to integrate the acquired skills in practice and adapt the techniques to different

235 children and contexts. As the training focused on simple HCTech, this allowed nurses to master

236 many different techniques. These results are unique in pediatrics, and are in line with studies

237 exploring prevention or alleviation of pain in adult patients that have used nurse-led hypnosis for burn-related pain $^{28}$ and gastrointestinal disorders ${ }^{29,30}$. 
240 demonstrated higher competencies in HCTech. Surprisingly, these differences were not associated

241 with nurses' initial motivation to take part in the training. When looking at these nurses' scores, it

242 would seem that the training has benefited them in different ways. Indeed, it seems as though the

243 training allowed nurse $\mathrm{C}$ to learn multiple new skills. It is possible that she was searching for

244 communication techniques prior to the study. On the other hand, it seems that the training allowed

245 nurse D to confirm the methods she was already intuitively using in her practice.

246 Importantly, despite the absence of complementary supervisions following the training

247 sessions, or further "booster" sessions, all newly acquired hypnotic communication skills

248 (relational and technical) were maintained over time at follow-up. Indeed, no statistically

249 significant difference was detected between post-training time-points and effect sizes were small.

250 Although this was not measured here, this may be due to a continuous practice of the techniques

251 learned. Continuous practice is an essential element of retention when acquiring new skills in

252 nursing education. ${ }^{31}$ This observation relates to what is observed in medical pedagogy, as noted by

253 Taylor Sawyer et al. ${ }^{32}$ It is possible that additional sessions may help participants improve

254 competencies after the core training. Future studies should focus on the effects of complementary

255 sessions or supervisions on learning and maintaining of hypnotic communication skills.

256 As for pertinence and acceptability, the feedback collected with nurses on this training 257 showed a mixed pattern. Although nurses positively assessed multiple components of the training,

258 such as role-play and the variety of techniques, participants highlighted limitations to the training.

259 Consequently, nurses offered suggestions for improvement of the training content and format, 260 including a more realistic setting. Such feedback is increasingly recognized as critical in the define-

261 refine phase of a new non-pharmacological intervention. ${ }^{33}$ Integrating these improvements in

262 future trainings and trials may further positively influence nurses' mastery of HCTech. 
264 limited in size due to the feasibility nature of the current study. It is probable that a larger sample

265 size would have allowed a greater variability in nurses' mastery of HCTech and offer more power

266 for inferential statistics. To deal with this issue we focused on effect sizes, following guidelines on

267 the development of non-pharmacological interventions. ${ }^{33}$ Yet, we adopted a strict design with the

268 same dyads being followed over time and the assessment of a high number (100+) of nurse-patient

269 interactions. Secondly, although we included two baseline time-points, this was too limited to

270 ascertain stability in the absence of training. Although this is improbable, changes over time in

271 communication behaviours may be due to other unmeasured factors. Similarly, we did not compare

272 changes with a control condition. Finally, sources of variance due to the different levels of data in

273 assessed interactions, i.e. nurses and patients, could not be explored with a formal multilevel

274 statistical design. Future studies should address these limitations including a control condition in a

275 larger sample allowing systematic multilevel analyses.

\section{5. Conclusion}

278 This study is the first to evaluate the training of pediatric nurses to hypnotic communication

279 techniques designed to manage pain and distress during medical procedures. Despite limitations,

280 changes occurred in nurses' communication behaviours in post-training with a clear improvement

281 in relational and technical skills, with changes being maintained over time. Participants offered

282 new ideas to improve the training. The present study is particularly original as it explicitly

283 evaluated change in nurses' practice. This opens a new field of research as future patient-level

284 outcomes could be attributed to actual changes in nurses' behaviours. The use of hypnotic

285 communication in hospital settings has the potential to benefit young patients' quality of life.

286 Future studies should systematically explore behavioural changes as a result of training, and 
287 hopefully demonstrate how this translates into patient-level outcomes such as procedural pain or 288 distress. 


\section{Acknowledgments}

The authors wish to thank Cybelle Abate for her help with the data collection.

$\underline{\text { Declaration of interests }}$

The authors declare no conflict of interests. 


\section{References}

1. Birnie KA, Noel M, Parker JA, et al. Systematic Review and Meta-Analysis of Distraction and Hypnosis for Needle-Related Pain and Distress in Children and Adolescents. Journal of Pediatric Psychology. 2014;39(8):783-808.

2. Kennedy RM, Luhmann J, Zempsky WT. Clinical implications of unmanaged needleinsertion pain and distress in children. Pediatrics. 2008;122:S130-S133.

3. Pai AL, Kazak AE. Pediatric medical traumatic stress in pediatric oncology: family systems interventions. Current Opinion in Pediatrics. 2006;18(5):558-562.

4. Rogers TL, Ostrow CL. The use of EMLA cream to decrease venipuncture pain in children. Journal of Pediatric Nursing. 2004;19(1):33-39.

5. Şahiner NC, İnal S, Akbay AS. The effect of combined stimulation of external cold and vibration during immunization on pain and anxiety levels in children. Journal of PeriAnesthesia Nursing. 2015;30(3):228-235.

6. Koller D, Goldman RD. Distraction Techniques for Children Undergoing Procedures: A Critical Review of Pediatric Research. Journal of Pediatric Nursing. 2012;27(6):652-681.

7. Accardi MC, Milling LS. The effectiveness of hypnosis for reducing procedure-related pain in children and adolescents: a comprehensive methodological review. Journal of behavioral medicine. 2009;32(4):328-339.

8. Kuttner L. A child in pain: what health professionals can do to help. Carmarthen, UK: Crown House Publishing; 2010. 
9. Hawkins PJ, Liossi C, Ewart BW, Hatira P, Kosmidis VH. Hypnosis in the alleviation of procedure related pain and distress in paediatric oncology patients. Contemporary Hypnosis. 1998;15(4):199-207.

10. Hilgard JR, Lebaron S. Relief of anxiety and pain in children and adolescents with cancer: Quantitative measures and clinical observations. International Journal of Clinical and Experimental Hypnosis. 1982;30(4):417-442.

11. Katz ER, Kellerman J, Ellenberg L. Hypnosis in the reduction of acute pain and distress in children with cancer. Journal of Pediatric Psychology. 1987;12(3):379-394.

12. Kuttner L, Bowman M, Teasdale M. Psychological Treatment of Distress, Pain, and Anxiety for Young Children with Cancer. Journal of Developmental and Behavioral Pediatrics. 1988;9(6):374-382.

13. Liossi C, Hatira P. Clinical hypnosis versus cognitive behavioral training for pain management with pediatric cancer patients undergoing bone marrow aspirations. International Journal of Clinical and Experimental Hypnosis. 1999;47(2):104-116.

14. Liossi C, Hatira P. Clinical hypnosis in the alleviation of procedure-related pain in pediatric oncology patients. International Journal of Clinical and Experimental Hypnosis. 2003;51(1):4-28.

15. Liossi C, White P, Hatira P. Randomized clinical trial of local anesthetic versus a combination of local anesthetic with self-hypnosis in the management of pediatric procedure-related pain. Health Psychology. 2006;25(3):307-315.

16. Liossi C, White P, Hatira P. A randomized clinical trial of a brief hypnosis intervention to control venepuncture-related pain of paediatric cancer patients. Pain. 2009;142(3):255-263. 
17. Smith JT, Barabasz A, Barabasz M. Comparison of hypnosis and distraction in severely ill children undergoing painful medical procedures. Journal of Counseling Psychology. 1996;43(2):187-195.

18. Wall VJ, Womack W. Hypnotic versus Active Cognitive Strategies for Alleviation of Procedural Distress in Pediatric Oncology Patients. American Journal of Clinical Hypnosis. 1989;31(3):181-191.

19. Zeltzer L, LeBaron S. Hypnosis and nonhypnotic techniques for reduction of pain and anxiety during painful procedures in children and adolescents with cancer. The Journal of Pediatrics. 1982;101(6):1032-1035.

20. Yeh VM, Schnur JB, Montgomery GH. Disseminating hypnosis to health care settings: Applying the RE-AIM framework. Psychology of Consciousness 2014;1(2):213-228.

21. Hammond DC. Handbook of hypnotic suggestions and metaphors. New York: WW Norton \& Company; 1990.

22. Kohen DP, Olness K. Hypnosis and hypnotherapy with children. Fourth ed. New York: Routledge; 2011.

23. Aramideh J, Mizrahi T, Charest M-C, Plante C, Duval M, Sultan S. Development and interrater reliability of a tool assessing hypnotic communication behaviours adopted by nurses caring for children with cancer: The Sainte-Justine Hypnotic Communication Assessment Scale. Complementary Therapies in Medicine. 2018;37:178-184.

24. Kazdin AE. Single-case research designs: Methods for clinical and applied settings. 2nd ed: New York, NY, US: Oxford University Press; 2011.

25. Cicchetti DV. Guidelines, criteria, and rules of thumb for evaluating normed and standardized assessment instruments in psychology. Psychological assessment. 1994;6(4):284-290. 
26. Braun V, Clarke V. Using thematic analysis in psychology. Qualitative research in psychology. 2006;3(2):77-101.

27. Wood C, Bioy A. Hypnosis and Pain in Children. Journal of Pain and Symptom Management. 2008;35(4):437-446.

28. Berger MM, Davadant M, Marin C, et al. Impact of a pain protocol including hypnosis in major burns. Burns. 2010;36(5):639-646.

29. Bremner H. Nurse-led hypnotherapy: An innovative approach to Irritable Bowel Syndrome. Complementary Therapies in Clinical Practice. 2013;19(3):147-152.

30. Smith GD. Effect of nurse-led gut-directed hypnotherapy upon health-related quality of life in patients with irritable bowel syndrome. Journal of Clinical Nursing. 2006;15(6):678684.

31. Oermann MH, Kardong-Edgren SE, Odom-Maryon T. Effects of monthly practice on nursing students' CPR psychomotor skill performance. Resuscitation. 2011;82(4):447-453.

32. Sawyer T, White M, Zaveri P, et al. Learn, See, Practice, Prove, Do, Maintain: An Evidence-Based Pedagogical Framework for Procedural Skill Training in Medicine. Academic Medicine. 2015;90(8):1025-1033.

33. Czajkowski SM, Powell LH, Adler N, et al. From ideas to efficacy: The ORBIT model for developing behavioral treatments for chronic diseases. Health Psychology. 2015;34(10):971-982. 
Figure 1. Flow of participants throughout the study
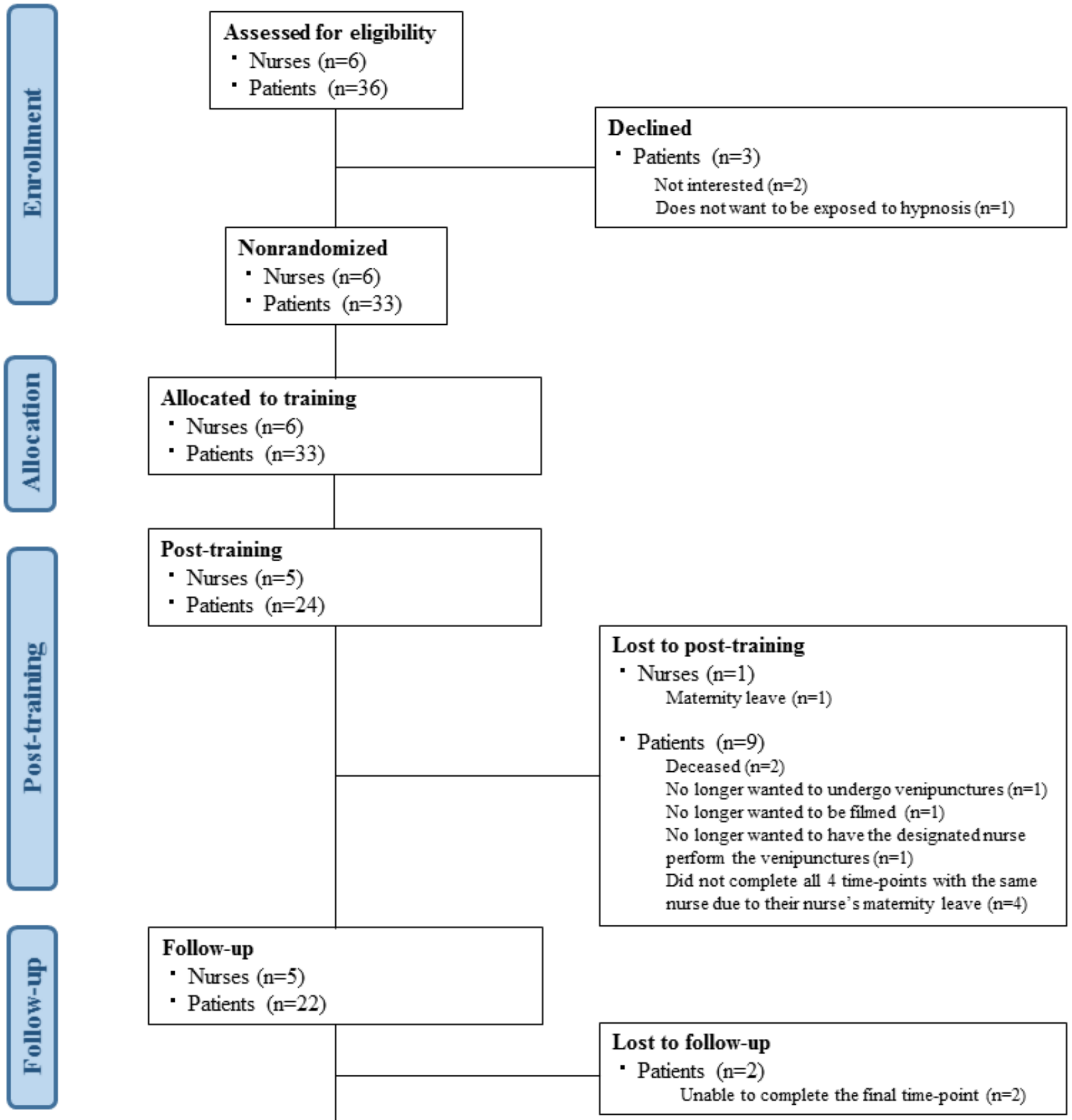

Analyzed for mastery of Hypnotic Communication

Post-training analyses

- Nurses ( $\mathrm{n}=5)$

- Patients ( $\mathrm{n}=24)$
Follow-up analyses

- Nurses ( $\mathrm{n}=5$ )

- Patients ( $\mathrm{n}=22)$ 
Table 1. Use of Hypnotic Communication Techniques by nurses performing venipunctures in a Hematology-Oncology daycare clinic, before and after training.

\begin{tabular}{|c|c|c|c|c|c|c|}
\hline & \multicolumn{2}{|c|}{ Baseline $^{\mathrm{a}}$} & \multicolumn{2}{|c|}{ Post-training $^{\mathrm{a}}$} & \multicolumn{2}{|c|}{ Follow-up ${ }^{a}$} \\
\hline & Nb of patients & $\mathrm{M}(\%)(\mathrm{SD})(\%)$ & Nb of patients & $\mathrm{M}(\%)(\mathrm{SD})(\%)$ & Nb of patients & $\mathrm{M}(\%)(\mathrm{SD})(\%)$ \\
\hline All nurses ${ }^{b}$ & $\mathrm{~N}=22$ & & $\mathrm{~N}=22$ & & $\mathrm{~N}=22$ & \\
\hline Total score & & $45.87(12.38)$ & & $59.92(14.21)^{* *}$ & & $59.09(13.67) * * *$ \\
\hline Relationship subscore & & $65.00(15.96)$ & & $77.27(9.35)^{* *}$ & & $75.45(10.57)^{* *}$ \\
\hline Technique subscore & & $29.93(12.51)$ & & $45.46(23.11)^{* *}$ & & $45.46(20.04)^{* *}$ \\
\hline Nurse A & $\mathrm{n}=5$ & & $n=5$ & & $\mathrm{n}=5$ & \\
\hline Total score & & $35.45(7.47)$ & & $49.09(13.79)$ & & $45.45(14.37)$ \\
\hline Relationship subscore & & $52.00(14.83)$ & & $68.00(17.89)$ & & $64.00(16.73)$ \\
\hline Technique subscore & & $21.67(7.45)$ & & $33.33(16.67)$ & & $30.00(13.94)$ \\
\hline Nurse B & $\mathrm{n}=5$ & & $\mathrm{n}=5$ & & $n=5$ & \\
\hline Total score & & $50.00(9.64)$ & & $54.55(0.00)$ & & $56.36(4.07)$ \\
\hline Relationship subscore & & $72.00(10.95)$ & & $80.00(0.00)$ & & $80.00(0.00)$ \\
\hline Technique subscore & & $31.67(13.69)$ & & $33.33(0.00)$ & & $36.67(7.45)$ \\
\hline Nurse C & $\mathrm{n}=3$ & & $\mathrm{n}=3$ & & $\mathrm{n}=3$ & \\
\hline Total score & & $31.82(9.09)$ & & $72.73(18.18)$ & & $63.64(15.75)$ \\
\hline Relationship subscore & & $46.67(11.55)$ & & $80.00(0.00)$ & & $73.33(11.55)$ \\
\hline Technique subscore & & $19.45(9.62)$ & & $66.67(33.33)$ & & $55.56(25.46)$ \\
\hline Nurse D & $\mathrm{n}=4$ & & $\mathrm{n}=4$ & & $\mathrm{n}=4$ & \\
\hline Total score & & $55.68(13.06)$ & & $72.73(7.42)$ & & $75.00(8.70)$ \\
\hline Relationship subscore & & $70.00(14.14)$ & & $80.00(0.00)$ & & $80.00(0.00)$ \\
\hline Technique subscore & & $43.75(12.50)$ & & $66.67(13.61)$ & & $70.83(15.96)$ \\
\hline Nurse F & $\mathrm{n}=5$ & & $\mathrm{n}=5$ & & $\mathrm{n}=5$ & \\
\hline Total score & & $52.73(5.18)$ & & $58.18(13.79)$ & & $60.00(8.13)$ \\
\hline Relationship subscore & & $78.00(4.47)$ & & $80.00(0.00)$ & & $80.00(0.00)$ \\
\hline Technique subscore & & $31.67(6.97)$ & & $40.00(25.28)$ & & $43.33(14.91)$ \\
\hline
\end{tabular}


Figure 2. Evolution of the use of hypnotic communication techniques over time and across nurses.

Fig. 2A. Total Score

Fig. 2B. Relationship subscore

Fig. 2C. Technique subscore
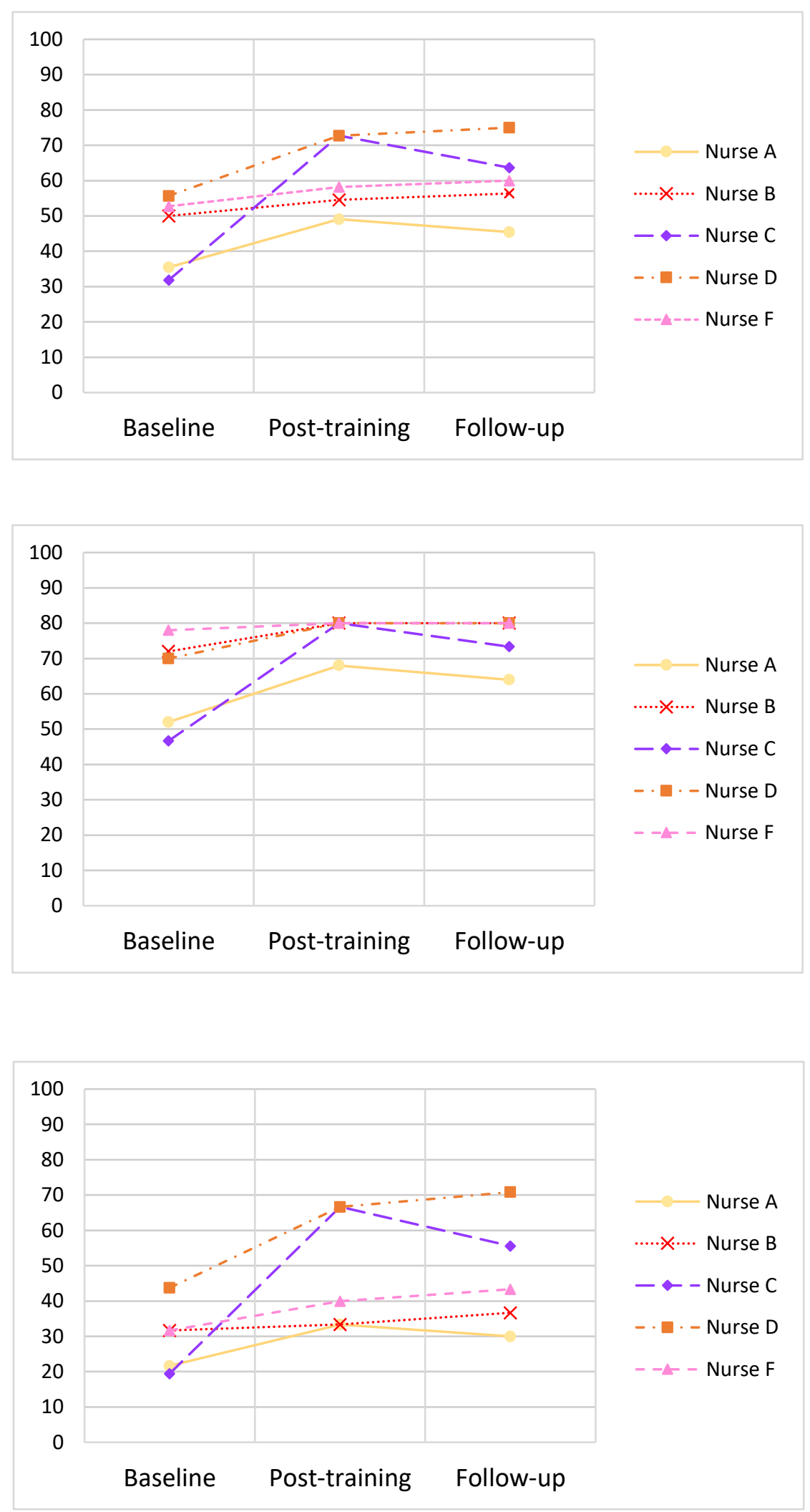
Figure 3. Cohen's $d$ effect size for baseline - post-training, baseline - follow-up, and post-training - follow-up comparisons for hypnotic communication techniques scores across nurses

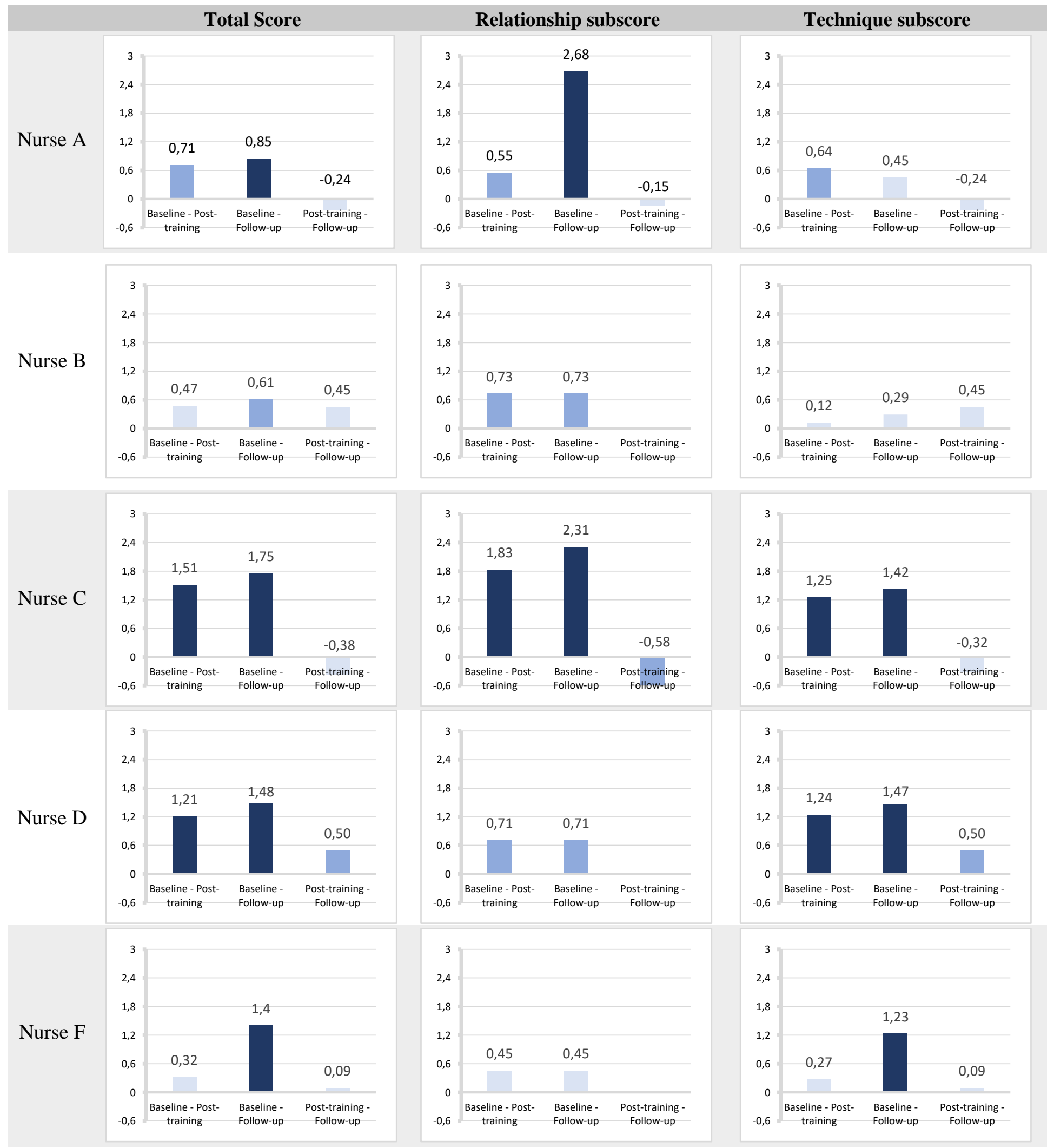

Effect sizes $(d)$ interpretation: $\quad$ small $(0.20-0.50)$, medium $(0.50-0.80)$, and $\square$ large $(0.80$ or higher $)$ 
Supplementary Table S1. Wilcoxon signed-rank non-parametric tests results and p values for the use of Hypnotic Communication Techniques by nurses performing venipunctures in a Hematology-Oncology clinic

\begin{tabular}{|c|c|c|c|}
\hline & Baseline - Post-training & Baseline - Follow-up & Post-training - Follow-up \\
\hline \multicolumn{4}{|l|}{ Nurses $^{\text {a }}$} \\
\hline Total score & $\mathrm{Z}=-3.138^{\mathrm{b}}, \mathrm{p}=0.002$ & $\mathrm{Z}=-3.614^{\mathrm{b}}, \mathrm{p}=0.000$ & $Z=-0.309^{c}, p=0.757$ \\
\hline Relationship subscore & $Z=-2.942^{b}, p=0.003$ & $\mathrm{Z}=-3.235^{\mathrm{b}}, \mathrm{p}=0.001$ & $Z=-0.707^{c}, p=0.480$ \\
\hline Technique subscore & $\mathrm{Z}=-2.710^{\mathrm{b}}, \mathrm{p}=0.007$ & $\mathrm{Z}=-2.976^{\mathrm{b}}, \mathrm{p}=0.003$ & $Z=-0.159^{b}, p=0.873$ \\
\hline \multicolumn{4}{|l|}{ Nurse A } \\
\hline Total score & $\mathrm{Z}=-1.225^{\mathrm{b}}, \mathrm{p}=0.221$ & $Z=-1.625^{b}, p=0.104$ & $Z=-0.577^{c}, p=0.564$ \\
\hline Relationship subscore & $\mathrm{Z}=-1.219^{\mathrm{b}}, \mathrm{p}=0.223$ & $\mathrm{Z}=-2.121^{\mathrm{b}}, \mathrm{p}=0.034$ & $Z=-0.378^{c}, p=0.705$ \\
\hline Technique subscore & $Z=-1.342^{b}, p=0.180$ & $Z=-0.921^{b}, p=0.357$ & $Z=-0.577^{c}, p=0.564$ \\
\hline \multicolumn{4}{|l|}{ Nurse B } \\
\hline Total score & $Z=-0.962^{b}, p=0.336$ & $Z=-1.219^{b}, p=0.223$ & $Z=-1.000^{b}, p=0.317$ \\
\hline Relationship subscore & $\mathrm{Z}=-1.414^{\mathrm{b}}, \mathrm{p}=0.157$ & $\mathrm{Z}=-1.414^{\mathrm{b}}, \mathrm{p}=0.157$ & $\mathrm{Z}=0.000^{\mathrm{d}}, \mathrm{p}=1.000$ \\
\hline Technique subscore & $Z=-0.276^{b}, p=0.783$ & $Z=-0.680^{b}, p=0.496$ & $\mathrm{Z}=-1.000^{\mathrm{b}}, \mathrm{p}=0.317$ \\
\hline \multicolumn{4}{|l|}{ Nurse C } \\
\hline Total score & $\mathrm{Z}=-1.826^{\mathrm{b}}, \mathrm{p}=0.068$ & $Z=-1.604^{b}, p=0.109$ & $\mathrm{Z}=-0.447^{\mathrm{c}}, \mathrm{p}=0.655$ \\
\hline Relationship subscore & $\mathrm{Z}=-1.841^{\mathrm{b}}, \mathrm{p}=0.066$ & $\mathrm{Z}=-1.633^{\mathrm{b}}, \mathrm{p}=0.102$ & $Z=-1.000^{c}, p=0.317$ \\
\hline Technique subscore & $\mathrm{Z}=-1.826^{\mathrm{b}}, \mathrm{p}=0.068$ & $Z=-1.604^{b}, p=0.109$ & $Z=-0.477^{c}, p=0.655$ \\
\hline \multicolumn{4}{|l|}{ Nurse D } \\
\hline Total score & $Z=-1.826^{b}, p=0.068$ & $Z=-1.841^{b}, p=0.066$ & $\mathrm{Z}=-1.000^{\mathrm{b}}, \mathrm{p}=0.317$ \\
\hline Relationship subscore & $\mathrm{Z}=-1.342^{\mathrm{b}}, \mathrm{p}=0.180$ & $\mathrm{Z}=-1.342^{\mathrm{b}}, \mathrm{p}=0.180$ & $\mathrm{Z}=0.000^{\mathrm{d}}, \mathrm{p}=1.000$ \\
\hline Technique subscore & $Z=-1.604^{b}, p=0.109$ & $Z=-1.633^{b}, p=0.102$ & $\mathrm{Z}=-1.000^{\mathrm{b}}, \mathrm{p}=0.317$ \\
\hline \multicolumn{4}{|l|}{ Nurse F } \\
\hline Total score & $Z=-0.535^{b}, p=0.593$ & $\mathrm{Z}=-1.841^{\mathrm{b}}, \mathrm{p}=0.066$ & $\mathrm{Z}=-0.272^{\mathrm{b}}, \mathrm{p}=0.785$ \\
\hline Relationship subscore & $\mathrm{Z}=-1.000^{\mathrm{b}}, \mathrm{p}=0.317$ & $\mathrm{Z}=-1.000^{\mathrm{b}}, \mathrm{p}=0.317$ & $\mathrm{Z}=0.000^{\mathrm{d}}, \mathrm{p}=1.000$ \\
\hline Technique subscore & $\mathrm{Z}=-0.535^{\mathrm{b}}, \mathrm{p}=0.593$ & $\mathrm{Z}=-1.841^{\mathrm{b}}, \mathrm{p}=0.066$ & $\mathrm{Z}=-0.272^{\mathrm{b}}, \mathrm{p}=0.785$ \\
\hline
\end{tabular}


Supplementary Table S2. Qualitative reporting of nurses' training assessment from semi-directive interviews

\begin{tabular}{|c|c|c|c|c|c|c|c|c|c|c|}
\hline & \multicolumn{2}{|c|}{ Motivation to participate } & \multirow{2}{*}{$\begin{array}{l}\text { Use of } \\
\text { techniques } \\
\text { in daily } \\
\text { practice }\end{array}$} & \multirow{2}{*}{$\begin{array}{l}\text { Changes in } \\
\text { practice }\end{array}$} & \multirow{2}{*}{$\begin{array}{l}\text { Patients' } \\
\text { and/or } \\
\text { families' } \\
\text { benefits }\end{array}$} & \multirow{2}{*}{$\begin{array}{l}\text { Personal } \\
\text { benefits }\end{array}$} & \multirow{2}{*}{$\begin{array}{l}\text { Training } \\
\text { satisfaction } \\
\text { (10-point } \\
\text { Likert } \\
\text { scale) }\end{array}$} & \multicolumn{2}{|c|}{ Training } & \multirow{2}{*}{ Recommended changes } \\
\hline & Reasons & $\begin{array}{l}\text { point } \\
\text { Likert } \\
\text { scale }\end{array}$ & & & & & & Positive aspects & Negative aspects & \\
\hline Nurse A & $\begin{array}{l}\text { Improve what } \\
\text { she was doing } \\
\text { with patients }\end{array}$ & 7 & Yes & $\begin{array}{l}\cdot \text { Yes, use of } \\
\text { «distraction». } \\
\cdot \text { Thinks about } \\
\text { what to say. }\end{array}$ & $\begin{array}{l}\text { Yes, more } \\
\text { pleasant and } \\
\text { easier. }\end{array}$ & · No & 5 & $\begin{array}{l}\text { Think about a } \\
\text { different } \\
\text { approaches }\end{array}$ & $\begin{array}{l}\text { Very theoretical } \\
\text { - Not clear how to } \\
\text { apply in daily } \\
\text { practice }\end{array}$ & $\begin{array}{l}\text { The training should be } \\
\text { more concrete (e.g. a nurse } \\
\text { with several people in a } \\
\text { room). }\end{array}$ \\
\hline Nurse B & Help patients & 5 & Yes & Not really & $\begin{array}{l}\text { No, not the } \\
\text { right } \\
\text { environment } \\
\text { (i.e. to } \\
\text { noisy) }\end{array}$ & - No & 5 & $\begin{array}{l}\text { Good } \\
\text { techniques }\end{array}$ & $\begin{array}{l}\cdot \text { Videos not } \\
\text { representative of } \\
\text { their reality } \\
\cdot \text { Targeting more } \\
\text { sensitive people } \\
\text { (nurses) }\end{array}$ & $\begin{array}{l}\text { The training should target } \\
\text { the nurses. }\end{array}$ \\
\hline Nurse C & $\begin{array}{l}\text { Curiosity to } \\
\text { learn } \\
\text { Believes in } \\
\text { hypnotic } \\
\text { communication }\end{array}$ & 10 & Yes & $\begin{array}{l}\text { Yes, realised } \\
\text { she was } \\
\text { already doing } \\
\text { it. More } \\
\text { «distraction» }\end{array}$ & $\begin{array}{l}\text { Yes, } \\
\text { decreases } \\
\text { anxiety. }\end{array}$ & $\cdot$ No & 8 & $\begin{array}{l}\text { Practice (in } \\
\text { training and in } \\
\text { the clinic) }\end{array}$ & $\begin{array}{l}\cdot \text { A lot of last } \\
\text { minute changes } \\
\cdot \text { Videos were not } \\
\text { related }\end{array}$ & $\begin{array}{l}\text { The training should } \\
\text { include more targeted videos } \\
\text { in a clinic illustrating the } \\
\text { technique (e.g. the } \\
\text { healthcare professional must } \\
\text { use the techniques). } \\
\text {. The training should focus } \\
\text { more on hypnotic } \\
\text { communication. }\end{array}$ \\
\hline Nurse D & $\begin{array}{l}\text { Clinic needs } \\
\text { Learn how to } \\
\text { improve pain } \\
\text { management }\end{array}$ & 6 & -Sometimes & $\cdot$ No & $\begin{array}{l}\text { Yes, some } \\
\text { techniques. }\end{array}$ & - No & 5 & $\begin{array}{l}\text { Tools and } \\
\text { ideas }\end{array}$ & $\begin{array}{l}\text { Less feasible } \\
\text { things (e.g. noisy } \\
\text { environment) }\end{array}$ & $\begin{array}{l}\text { The training should better } \\
\text { understand the clinical } \\
\text { reality. } \\
\text { The training should be } \\
\text { adapted to nurses' needs. }\end{array}$ \\
\hline Nurse F & $\begin{array}{l}\text { Curiosity } \\
\text { - Learn ways to } \\
\text { help reduce pain }\end{array}$ & 9 & Yes & $\begin{array}{l}\text { Yes, at } \\
\text { times. Other } \\
\text { ways to } \\
\text { interact and } \\
\text { change } \\
\text { patients' } \\
\text { focus. }\end{array}$ & $\begin{array}{l}\text { Yes, } \\
\text { decreases } \\
\text { anxiety. }\end{array}$ & $\begin{array}{l}\text { Yes, } \\
\text { decreases } \\
\text { stress. }\end{array}$ & 7 & $\begin{array}{l}\text { Role play and } \\
\text { examples } \\
\text { (provided } \\
\text { documents) }\end{array}$ & $\begin{array}{l}\text { Videos not } \\
\text { representative } \\
\text { - Practice } \\
\text { techniques with } \\
\text { other medical } \\
\text { procedures to } \\
\text { considering a lot of } \\
\text { concentration is } \\
\text { needed for VPs }\end{array}$ & $\begin{array}{l}\text { The training should } \\
\text { include videos that represent } \\
\text { the clinical reality. }\end{array}$ \\
\hline
\end{tabular}


Supplementary Table S3. Full available data including drop-outs on the use of Hypnotic Communication Techniques by nurses performing venipunctures in a Hematology-Oncology clinic, before and after training.

\begin{tabular}{|c|c|c|c|c|c|c|}
\hline & \multicolumn{2}{|c|}{ Baseline } & \multicolumn{2}{|c|}{ Post-training } & \multicolumn{2}{|c|}{ Follow-up } \\
\hline & Nb of patients & $\mathrm{M}(\%)(\mathrm{SD})(\%)$ & Nb of patients ${ }^{c}$ & $\mathrm{M}(\%)(\mathrm{SD})(\%)$ & Nb of patients ${ }^{d}$ & $\mathrm{M}(\%)(\mathrm{SD})(\%)$ \\
\hline All nurses ${ }^{a}$ & $\mathrm{~N}=33$ & & $\mathrm{~N}=24$ & & $\mathrm{~N}=22$ & \\
\hline Total score & & $47.80(11.87)$ & & $60.23(13.88)$ & & $59.09(13.67)$ \\
\hline Technique subscore & & $32.32(11.74)$ & & $45.83(22.66)$ & & $45.46(20.04)$ \\
\hline Nurse A & $n=6$ & & $\mathrm{n}=6$ & & $\mathrm{n}=5$ & \\
\hline Total score & & $38.64(10.27)$ & & $50.00(12.53)$ & & $45.45(14.37)$ \\
\hline Nurse B & $\mathrm{n}=5$ & & $\mathrm{n}=5$ & & $\mathrm{n}=5$ & \\
\hline Total score & & $50.00(9.64)$ & & $54.55(0.00)$ & & $56.36(4.07)$ \\
\hline Relationship subscore & & $72.00(10.95)$ & & $80.00(0.00)$ & & $80.00(0.00)$ \\
\hline Technique subscore & & $31.67(13.69)$ & & $33.33(0.00)$ & & $36.67(7.45)$ \\
\hline Nurse $\mathbf{C}$ & $n=6$ & & $n=4$ & & $\mathrm{n}=3$ & \\
\hline Total score & & $35.61(9.28)$ & & $72.73(14.85)$ & & $63.64(15.75)$ \\
\hline Technique subscore & & $43.44(10.87)$ & & $66.67(13.61)$ & & $70.83(15.96)$ \\
\hline Nurse $\mathbf{E}^{\mathbf{b}}$ & $\mathrm{n}=5$ & & & & & \\
\hline Total score & & $57.27(4.07)$ & & & & \\
\hline Relationship subscore & & $80.00(12.25)$ & & & & \\
\hline Technique subscore & & $38.33(9.50)$ & & & & \\
\hline Nurse F & $\mathrm{n}=6$ & & $\mathrm{n}=5$ & & $\mathrm{n}=5$ & \\
\hline Total score & & $54.55(6.43)$ & & $58.18(13.79)$ & & $60.00(8.13)$ \\
\hline Relationship subscore & & $78.33(4.08)$ & & $80.00(0.00)$ & & $80.00(0.00)$ \\
\hline Technique subscore & & $34.72(9.74)$ & & $40.00(25.28)$ & & $43.44(14.91)$ \\
\hline
\end{tabular}




\section{Hypnotic communication techniques}

This supplementary file is taken from: Aramideh, J., Mizrahi, T., Charest, M.-C., Plante, C., Duval, M., \& Sultan, S. (2018). Development and inter-rater reliability of a tool assessing hypnotic communication behaviours adopted by nurses caring for children with cancer: The Sainte-Justine Hypnotic Communication

Assessment Scale. Complementary Therapies in Medicine, 37, 178-184. https://doi.org/10.1016/j.ctim.2017.11.013

Techniques

Switch box

\section{Numbness and Changes in Perception}

\section{Definitions and Examples}

"First, pay attention to your hand. Notice how you can feel tingling feelings in that hand. Then let it become numb. When it is very numb, touch that hand to your jaw (or other body part) and let the numb feeling transfer from the hand to the jaw." (Kohen \& Olness, 2011)
"The therapist explains the idea that pain is transmitted by nerves from various parts of the body to the brain, which then sends a pain message back to the body. The therapist can describe nerves and their pathways or can ask the child to provide a colour for nerves. The importance of accuracy varies with the age and needs of the child. The child is then asked to choose some sort of switch that can turn off incoming nerve signals. The therapist can describe various kinds of switches, such as flip, dimmer, pull or even a television computer push-button panel or control panel of lights. Having chosen a switch, the child is asked to begin practicing turning off the switches or the lights that connect the brain and certain areas of the body. It is useful to ask the child to turn off the incoming nerve signals for defined periods of time (e.g., 10 minutes, 15 minutes, 90 minutes). The success of the exercise is judged by touching the child with a small-gauge needle or some other sharp object and asking for a comparison with feelings on the other side where the nerve signals are unchanged." (Kohen \& Olness, 2011)

"Request for numbness": "You know what a numb feeling is. How does numbness feel to you?" Child responds. "Good, just let that part of your body get numb now. Numb like a block of ice (or whatever image the child has used)." (Kohen \& Olness, 2011)

"Topical anesthesia": "Just imagine painting numbing medicine onto that part of your body. Tell me when you're finished doing that." (Kohen \& Olness, 2011)

"Local anesthesia": "Imagine putting an anesthetic into that part of your body. Feel it flow into your body and notice the change in feeling as the area becomes numb." (Kohen \& Olness, 2011)

\section{Guided Imagery}

"Cognitive-behavioural intervention defined as concentrated focusing on images formed in the mind, through which the patient is helped to relax, focus, and develop mental images that result in the alteration of perceived pain or distress." (Kohen \& Olness, 2011) 
Deep breathing: bubble, Example: Bubble party blowers...

- $\quad$ "Capturing the attention of a small child, offering him to blow bubbles. The child applies himself to blow, to make the bubble travel; the breathing exercise brings him relaxation, the bubble's travel takes him away from the unpleasant act that we are doing to him and distracts him, he forgets that we are pricking him and that we are restraining him." (AREMIG, 2014)

Conversational Hypnosis or Covert Hypnosis
"Conversational hypnosis, also known as covert hypnosis, is a way of communicating with patients' unconscious without informing them. In this approach, the hypnotherapist slowly sends hypnotic messages to the patient and reduces the patient's resistance to alter his/ her thoughts, emotions, and beliefs." (Izanloo \& al., 2015)

Examples from Hypnosis and pain in children (Wood \& Bioy, 2008)

- Projecting the patient into the future of a procedure: "How happy you will be once I finish my clinical exam when you can watch the TV."

- When writing the medical prescription: "I'm going to prescribe this drug for you... and you will be surprised to notice that not only your pain is improved...but that your sleep is getting better." 


\section{References}

Aramideh, J., Mizrahi, T., Charest, M.-C., Plante, C., Duval, M., \& Sultan, S. (2018). Development and inter-rater reliability of a tool assessing hypnotic communication behaviours adopted by nurses caring for children with cancer: The Sainte-Justine Hypnotic Communication Assessment Scale. Complementary Therapies in Medicine, 37, $178-184$. doi:https://doi.org/10.1016/j.ctim.2017.11.013

Association pour la Recherche et les Études dans les Maladies Infantiles Graves (AREMIG) (2014). Témoignages sur la formation en Hypnoanalgésie. Retrieved from www.aremig.org/wp-content/uploads/2014/06/hypnoanalgesie.pdf

Kohen, D. P., \& Olness, K. (2011). Hypnosis and hypnotherapy with children (Fourth ed.). New York: Routledge.

Izanloo, A., Fathi, M., Izanloo, S., Vosooghinia, H., Hashemian, A., Sadrzadeh, S. M., \& Ghaffarzadehgan, K. (2015). Efficacy of Conversational Hypnosis and Propofol in Reducing Adverse Effects of Endoscopy. Anesthesiology and Pain Medicine, 5(5), e27695. doi:10.5812/aapm.27695

Wood, C., \& Bioy, A. (2008). Hypnosis and pain in children. Journal of pain and symptom management, 35(4), 437446. 